\title{
Experimental study of water drop motions induced by superposition of vibrations and shear flows
}

\author{
T. Maurer \& U. Janoske \\ Chair of Fluid Mechanics, University of Wuppertal, Germany
}

\begin{abstract}
Droplet motion induced by mechanical vibrations and superposed shear flows has not yet been investigated in detail, despite its major importance in many applications. The current study introduces a novel experimental setup for this purpose. Furthermore, droplet motion measurements on acrylic glass surfaces are presented. This study observes mechanical vibrations and shear flows separately as well as in combination. Measurements with sinusoidal vibrations show that the droplets overcome the force due to contact angle hysteresis, when vibrational acceleration is increased above a certain threshold. Consequently, droplets experience a lateral motion, particularly if the vibrational frequency matches their natural frequency. Separate experiments with shear flows indicate that droplet motions are initiated when a critical flow velocity is reached. The flow velocity required to initiate drop motions decreases when increasing droplet volume. Superposing vibration with the shear flow results in an inconvenient droplet contour and motion, the shaking slip motion. Additionally, the required flow velocity for droplet motion reduces noticeably. Furthermore, the influence of vibrations on the initial droplet motion increases with higher droplet volume. The study concludes that the mobility of water droplets on acrylic glass surfaces increases significantly when a superposition between vibrational forces and shear flows exists.

Keywords: droplet, vibration, shear flow, contact angle hysteresis, contact line, wetting phenomena.
\end{abstract}




\section{Introduction}

The concept of droplet transport driven by external forces is versatile and has attracted great interest in recent years. Droplet motion has great importance in practical applications such as heat transfer, microfluid devices or process industries. The motion of droplets is effected in different ways such as oscillating pressure fields, dielectric potential, vibrational forces or shear flows. In the present work we focus on the vibrational force and shear flow as well as the superposition of both.

Observations of droplet motion due to harmonic oscillations are reported by Daniel and Chaudhury [1]. The authors observed that a sessile drop on a gradient surface overcomes contact angle hysteresis if a periodic force is applied. Moreover, a droplet below a radius of $r=0.55 \mathrm{~mm}$ does not move, when the driving force due to free energy gradient is balanced by contact angle hysteresis. In the next step, Daniel et al. [2] investigated a sessile drop on a substrate when subjected to asymmetric vibration. The drop drifts on a chemically heterogeneous surface. Brunet et al. [3] and Noblin et al. [4] also considered droplet behavior due to asymmetric vibrational excitation. They observed that a droplet can not only overcome contact angle hysteresis due to vibration, moreover it overcomes gravity and the droplet starts climbing on an inclined plane. This is only possible above a certain vibrational acceleration threshold [3]. Shastry et al. [5] show the behavior of droplets on microstructured surfaces. Consequently, the drop moves down the gradient when vibration forces overcome contact angle hysteresis. The majority of the experiments concerning droplet moving due to vibrational force are observed on surfaces with gradients [6-8]. In this case the moving direction of the droplet is predetermined. In this study the droplet surface has no defined gradient and we assume that the acrylic glass surface is homogeneous.

Because of their importance and industrial relevance, the resonant frequencies of sessile drops have been studied intensively. Basic results for the oscillation of free liquid drops are presented by Lamb [9]. The author ignored the viscous drop damping and presented a general expression for different vibration modes:

$$
\omega=\sqrt{\frac{\gamma}{3 \pi m} l(l-1)(l+2)} .
$$

Where $\omega$ is the resonant frequency, $l$ an integer value of 2 or higher, $\gamma$ and $m$ are the surface tension and mass of the drop, respectively. Further results are presented by a multiplicity of authors, such as Lyubimov et al. [10], Celestini and Kofman [11] and Moon et al. [12]. In the present study the natural frequencies are calculated by eqn. (1).

The effect of droplet motion due to shear flows has received less attention than the influence of vibration. For two fluid phases three possible combinations exist, which can be arranged in the following categories: (a) a gas bubble displaced by a continuous liquid phase; (b) a liquid droplet displaced by a continuous liquid phase; (c) a liquid droplet displaced by a continuous gas phase. Within the scope 
of the present study we investigate the displacement of a droplet by a continuous gas phase.

The displacement of gas bubbles by a continuous liquid phase receives interest in applications such as boiling of liquids. An important contribution belongs to the buoyancy of the bubble. Al-Hayes and Winterton [13] balanced buoyancy, drag and surface tension forces. To correct the drag coefficient they used experimental data. They obtained a semiempirical prediction of the maximum bubble diameter. More recently, Duhar and Colin [14] used a similar force balance approach and included additional terms into the expression for drag force. With a set of experiments they determined a typical value for the contact angle that the bubble makes with the surface at the point of detachment.

A continuous liquid phase displacing a liquid droplet has been studied on smooth as well as rough surfaces. Detachment of a liquid drop from a porous surface by shear flow is numerically observed by Theodorakakos et al. [15]. The computational model includes contact angle hysteresis and shows physically correct behavior of the droplet.

A criterion for initiation of droplet movement of a liquid droplet displaced by a gas phase is introduced by Durbin [16]. The author balances the pressure due to air flow over the droplet with the surface tension forces that act to minimize droplet deformation. The model demonstrated that the air velocity required to initiate droplet motion depends strongly on contact angle hysteresis. Recently Fan et al. [17] postulated a model that balances surface tension forces at the contact line and the drag force due to air motion. The results show, that the initiation of droplet motion depends on the contact angle and the droplet size.

To the best of the authors knowledge there are no studies regarding the droplet motion due to superposition of harmonic oscillation and shear flows.

This paper considers the influence of vibration and shear flow separately as well as in combination. The main objective is to study the effect on the droplet motion induced by the superposition of vibrations and shear flows. We present a novel experimental setup which allows the study of droplet motion with vibrations and shear flows separately as well as in combination.

\section{Experimental setup}

The apparatus designed and realized within the scope of this study is shown schematically in fig. 1 . The experimental setup features three main parts: droplet generation and positioning, vibration as well as the shear flow generation and an imaging system.

A microsyringe is used to generate water droplets of different sizes $(5-12 \mu \mathrm{l})$. The accuracy of the droplet generation is verified by means of an analytical balance (Sartorius, CPA225D). Droplet weight measurements are conducted twenty times for every investigated droplet size. The results show a very good reproducibility of the droplet size with an averaged standard deviation of $\sigma=0.02 \mu \mathrm{l}$. To ensure repeatability in placement, the droplet is formed on the tip of the syringe and gently lowered onto the surface of the acrylic channel using an elevating screw. This 


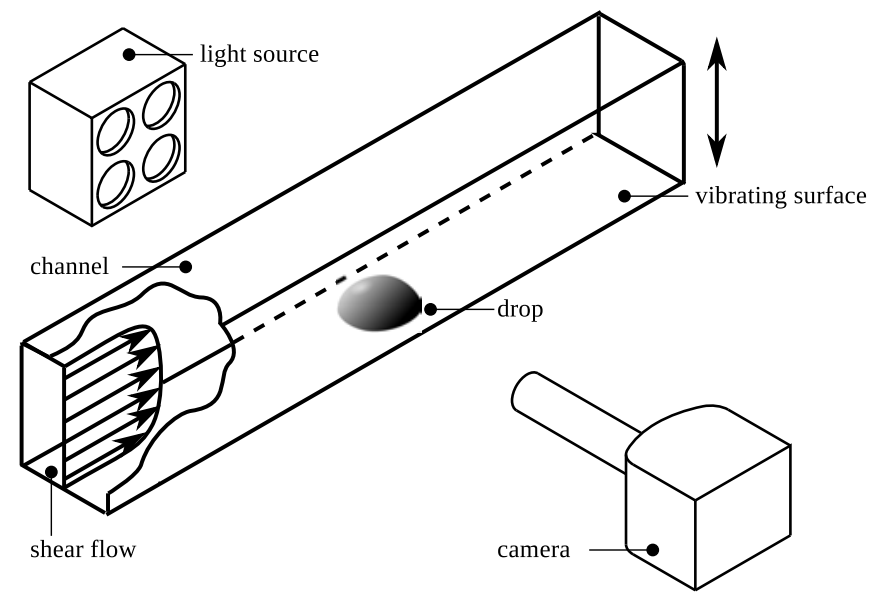

Figure 1: Schematical experimental setup.

channel has a width of $22 \mathrm{~mm}$ and a variable height ranging between $15-30 \mathrm{~mm}$. Hence, it is possible to realize Reynolds numbers from Re $=1000-45000$. In this study turbulent flow regimes $(\mathrm{Re}=6000-16000)$ are investigated.

The acrylic surface of the channel is placed on an electromagnetic shaker (Bruel and Kjær, V455), which constitutes the main part of the vibration system (fig. 2). A sinusoidal signal is generated by a frequency generator and enhanced in an amplifier. The amplifier directs the signal to the shaker and the acrylic substrate with the droplet experiences a sinusoidal motion. This motion is controlled and monitored by two acceleration sensors which are connected to a computer. With this vibrational system it is possible to realize vibrational accelerations up to $300 \mathrm{~m} / \mathrm{s}^{2}$. The shear flow is controlled by a mass flow controller (Bürkert, Type 8626). The flow exits the mass flow controller and enters a tube which directs the flow through a honeycomb into the channel and the measurement area.

To ensure good image quality in the observed ares, the droplet is illuminated by a strong light source from the back. Additionally the image is recorded with a high-speed camera and a macro-objective from the front. The observed area $(15 \times 5 \mathrm{~mm})$ includes a total amount of $1580 \mathrm{px} \times 580 \mathrm{px}$. Depending on the channel velocity, a frame rate between $500 \mathrm{f} / \mathrm{s}$ and $5000 \mathrm{f} / \mathrm{s}$ is chosen. Postprocessing of the measurement data is achieved with the open source software DropSnake. DropSnake is a spline-based approach for accurate determination of contact points and contact angles. Mirror symmetry allows detection of the position of the contact points, using the reflection of the drop at the substrate. In this study, this approach is also partially applied.

This novel experimental setup allows the superposition of a vibrational excitement and a shear flow on a droplet. Fig. 2 shows a photo of the final experimental realization. 


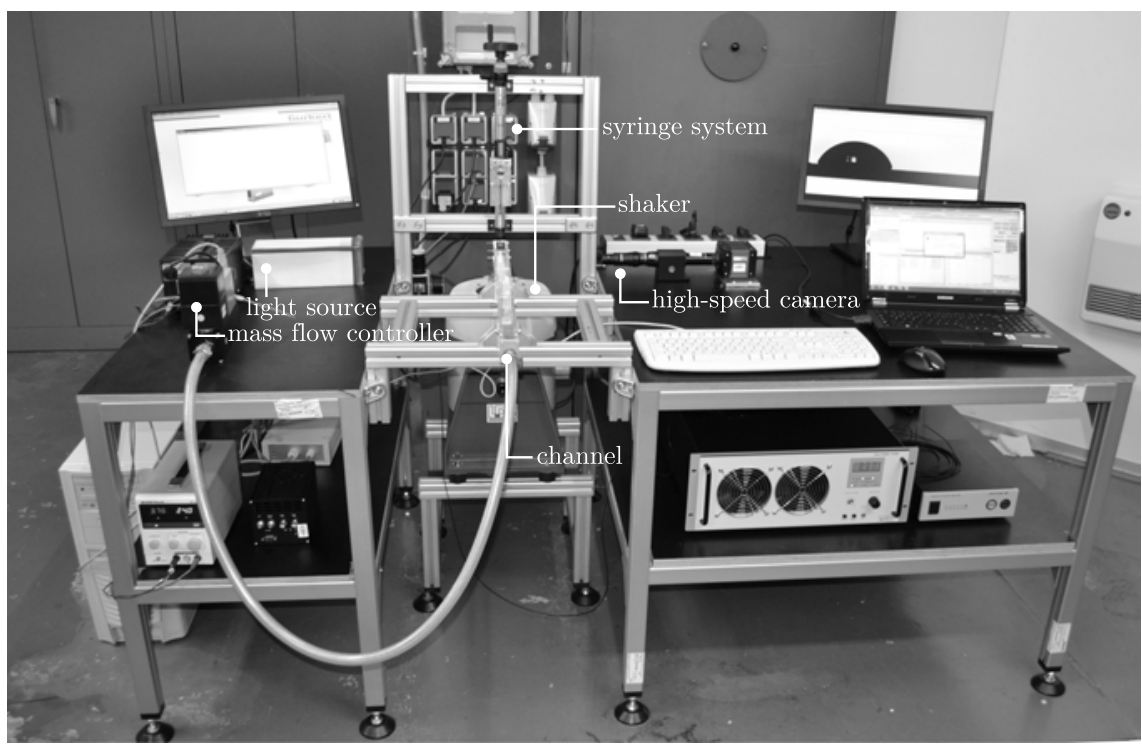

Figure 2: Realization of the experimental setup.

\section{Results}

We present the results of our study in terms of the critical Reynolds number $\mathrm{Re}_{\text {crit }}$. This number describes the droplet motion with and without the addition of vibrational excitation. $\mathrm{Re}_{\text {crit }}$ is defined as

$$
\operatorname{Re}_{\text {crit }}=\frac{v_{c h} \cdot d_{h}}{\nu},
$$

where $v_{c h}$ is the mean channel velocity, $d_{h}$ the hydraulic diameter of the channel and $\nu$ the kinematic viscosity of air. In all subsequent experiments the hydraulic diameter is $d_{h}=17.8 \mathrm{~mm}$ and the kinematic viscosity is $\nu=1.5 \cdot 10^{-5} \mathrm{~m}^{2} / \mathrm{s}$.

\subsection{Vibration experiments}

In this section the threshold of the vibrational acceleration between droplet motion and droplet sticking is presented. The acceleration is given by

$$
a=(2 \pi f)^{2} A,
$$

where $A$ and $f$ are the amplitude and the frequency of the applied vibration, respectively. The frequency $f$ is set to the resonant frequency of the drop which is calculated by eqn. (1). All experiments are conducted with the frequencies of the 1st Rayleigh mode. Table 1 shows the frequencies used.

For every droplet size we start with an acceleration of $20 \mathrm{~m} / \mathrm{s}^{2}$ which is increased slowly, until a droplet motion can be observed. If a motion is 
detected, we repeat the measurements up to this vibrational acceleration ten times. Additionally, measurements with an acceleration slightly below the threshold value are conducted. Such, if no droplet motion is observed, we can ensure that we found the acceleration threshold.

Fig. 3 shows that an increase of the droplet volume leads to a smaller acceleration threshold. In other words, a smaller drop needs higher vibrational force to overcome contact angle hysteresis, because of the smaller inertia. Furthermore, fig. 3 indicates that above a vibrational acceleration of approximately $182 \mathrm{~m} / \mathrm{s}^{2}$ the drop starts to move independently of the droplet size. Sticking is observed below a vibrational acceleration about $112 \mathrm{~m} / \mathrm{s}^{2}$. To investigate the contribution of the vibrational excitation to the critical Reynolds number, we use the sticking regime below $112 \mathrm{~m} / \mathrm{s}^{2}$ for the superposed case. Thus, the droplet motion for the superposed case is increased by but not initiated due to vibrational acceleration.

Table 1: 1st Rayleigh modes for different droplet sizes.

\begin{tabular}{|l||c|c|c|c|c|}
\hline Droplet volume & $5 \mu \mathrm{l}$ & $6 \mu \mathrm{l}$ & $9 \mu \mathrm{l}$ & $10 \mu \mathrm{l}$ & $12 \mu \mathrm{l}$ \\
\hline 1st Rayleigh mode $\omega$ & $110.6 \mathrm{~Hz}$ & $100.9 \mathrm{~Hz}$ & $82.4 \mathrm{~Hz}$ & $78.2 \mathrm{~Hz}$ & $71.4 \mathrm{~Hz}$ \\
\hline
\end{tabular}

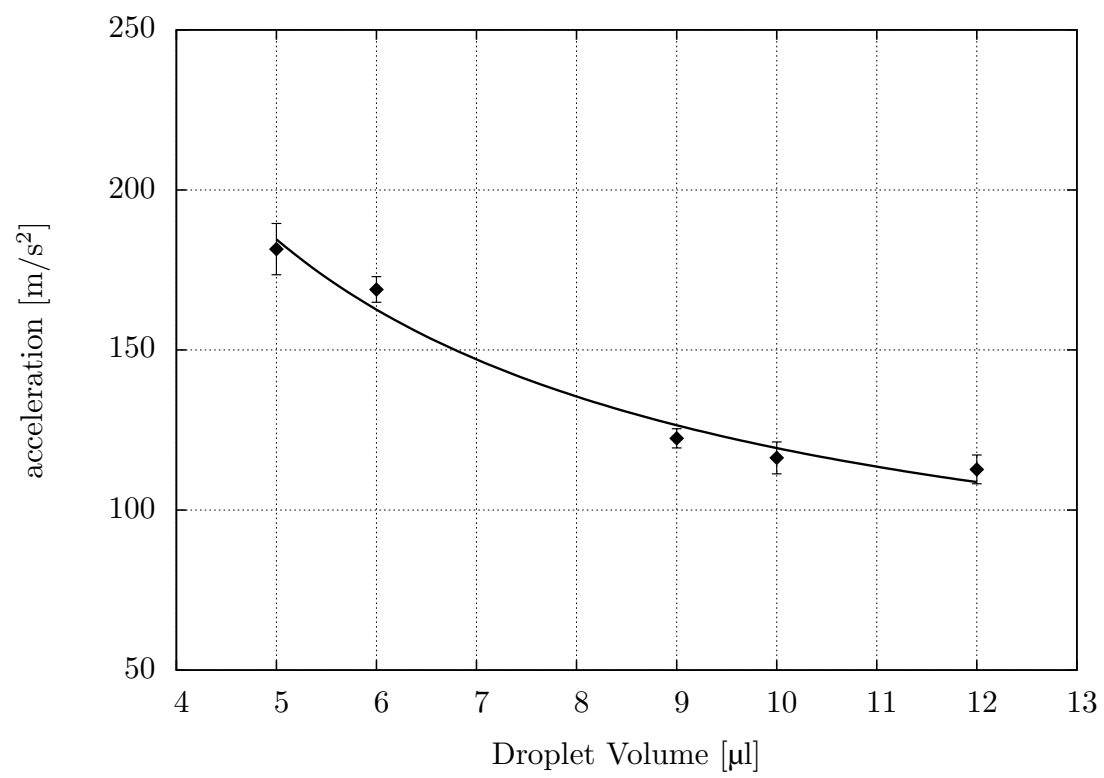

Figure 3: Vibrational acceleration threshold for different droplet volumes. 

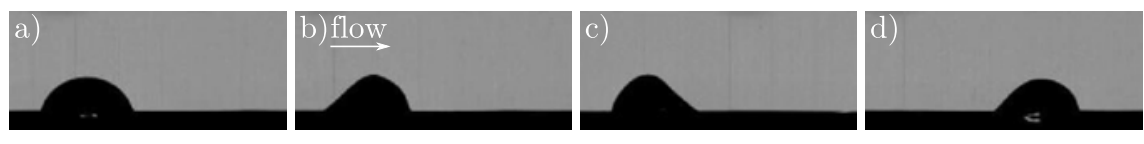

Figure 4: Droplet contour due to shear flow.

\subsection{Shear flow experiments}

The influence of shear flow on the motion of droplet is investigated. The critical Reynolds number when the drop starts to move is used as an reference value for the superposed case. We obtain this reference value by increasing the channel velocity step by step until a droplet motion is observed.

Before the shear flow reaches the critical velocity the drop sticks on the acrylic glass surface and shows a forward-backward oscillation behavior. This phenomenon, illustrated in fig. 4 , is a result of the interaction between the surface tension, surface roughness, shear flow and liquid properties of water. Without the shear flow neither a droplet oscillation nor a droplet motion can be observed (fig. 4a). With the shear flow a forward motion of the droplet is induced by inertia until a critical forward position (fig. $4 \mathrm{~b}$ ) is reached. At this point the kinetic energy gains its maximum value. This is followed by a backward motion initiated by viscous effects until a critical backward position (fig. 4c) is achieved. At this point the kinetic energy is zero and starts to increase again. This is the beginning of the next oscillating period. The channel velocity (thus the Reynolds number) is increased, until the droplet starts a slip motion downwards the flow direction (fig. 4d).

Fig. 5 shows the Reynolds number needed to initiate droplet motion as a function of the droplet size. It can be seen, that higher droplet volumes lead to smaller critical Reynolds numbers. Fan et al. [17] verified a similar behavior for different substrate-liquid combinations. Moreover, this correlation can be explained by two contribution factors.

The droplet sits within the laminar sub-layer and experiences a velocity gradient. By increasing the droplet size the distance to the surface also increases. Consequently, the droplet sees a higher mean air velocity and starts to move. Furthermore, inertia increases with higher drop volume although the contact line and therefore the sticking area are also rising.

\subsection{Superposition of vibration and shear flow}

The interaction between shear flow and vibrational acceleration leads to an inconvenient droplet contour and motion as can be seen in fig. 6 .

At the beginning of an oscillating period (fig. 6a) the droplet contour looks familiar to the forward position of the sticking motion (fig 4b). A little dent at the advancing area, which is caused by the shaking motion, separates these droplet contours. At the highest elevation (fig. 6b) the droplet shows an asymmetric and 


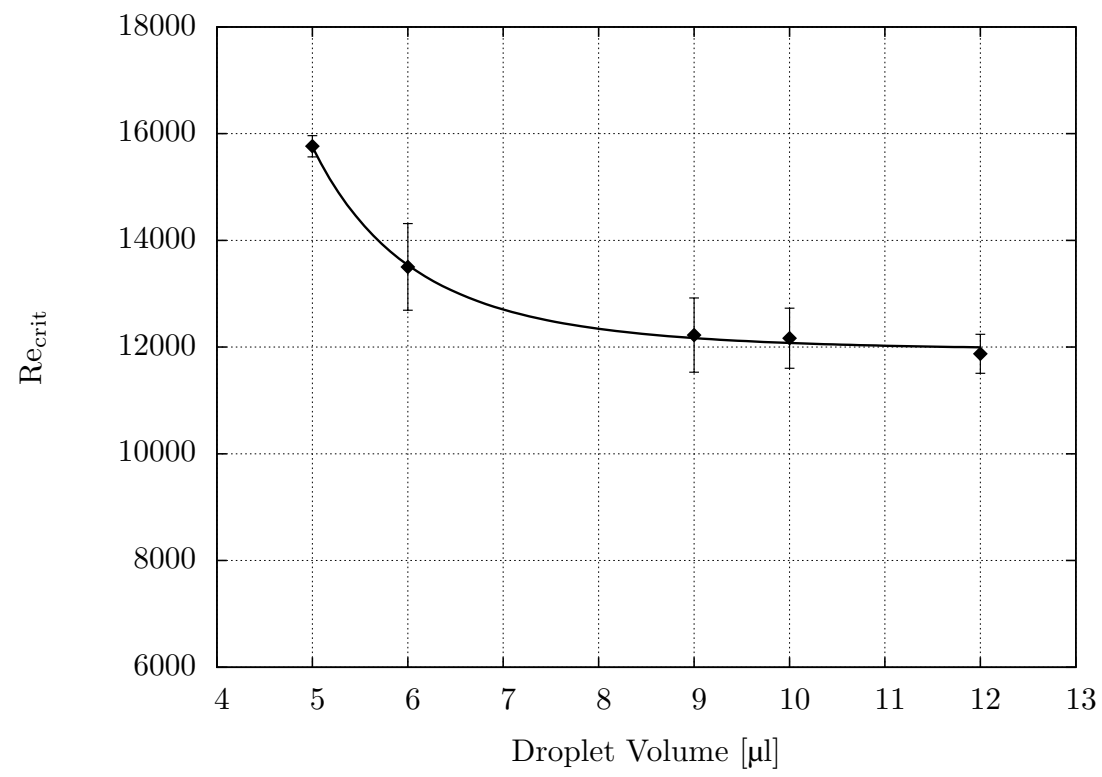

Figure 5: Reynolds number due to shear flow for different droplet volumes.

very flat contour. Here, the shaking motion dominates the viscous effects of the droplet. After the zero position the surface reaches the lowest elevation (fig. 6c). Here, a strong contour dent as well as noticeable slip motion are observed. The contour dent results from the downward surface motion. The lower droplet part experiences a downward motion due to vibration and adhesion force, whereas the fluid viscosity tights the upper part towards the surface. A contour peak occurs and the shear flow sees a higher obstacle which results in a downstream slip motion of the droplet. Proceeding to the zero position (fig. 6d) the dent becomes smaller and expands. The lower droplet part is moving upwards, whereas the upper part still moves towards the acrylic glass surface. Afterwards the next vibration period begins. Since the shaking contributes significantly to the droplet slipping, we call this motion the shaking slip motion of a droplet.

The critical Reynolds number for the shaking slip motion is also investigated. Fig. 7 shows the impact of harmonic oscillations on the critical Reynolds number. With a vibrational acceleration of $\mathrm{a}=50 \mathrm{~m} / \mathrm{s}^{2} \mathrm{Re}_{\text {crit }}$ reduces on average by $33 \%$. Increasing the acceleration to $\mathrm{a}=100 \mathrm{~m} / \mathrm{s}^{2}$ leads to an average reduction of the critical Reynolds number by $41 \%$ compared to the experiments without vibrations (fig. 5). In both experiments the influence of vibrations on the initial droplet motion increases with higher droplet volume. Furthermore, the results indicate that a threshold for the critical Reynolds number exists at approximately $\operatorname{Re}_{\text {crit }}=6900$. To confirm this, a wider range of droplet sizes and other surface-fluid combinations are necessary. 
It can be seen that the mobility of water droplets on acrylic glass surfaces increases significantly when a superposition between vibrational forces and shear flows exists. Additionally, an inconvenient shaking slip motion of the drop is observed.

\section{Conclusion}

The present study introduces an experimental setup for the investigation of water droplet motion due to mechanical vibrations and superposed shear flows. Furthermore, droplet motion measurements on acrylic glass surfaces are presented. The vibrations are generated by an electromagnetic shaker. Shear flows over the water droplet $(5 \mu \mathrm{l}-12 \mu \mathrm{l})$, which is positioned in a rectangular channel with a variable cross section, are controlled and monitored by a mass flow controller. To visualize droplet motions a diffused back light in combination with a high-speed camera is used.

This study considers mechanical vibrations and shear flows separately as well as in combination. Measurements with sinusoidal vibrations confirm that a droplet overcomes the force due to contact angle hysteresis when vibrational acceleration is increased above $182 \mathrm{~m} / \mathrm{s}^{2}$. The droplet experiences a lateral motion, particularly if the vibrational frequency matches the natural frequency. For any droplet size, a droplet remains sticking with an acceleration below $112 \mathrm{~m} / \mathrm{s}^{2}$.

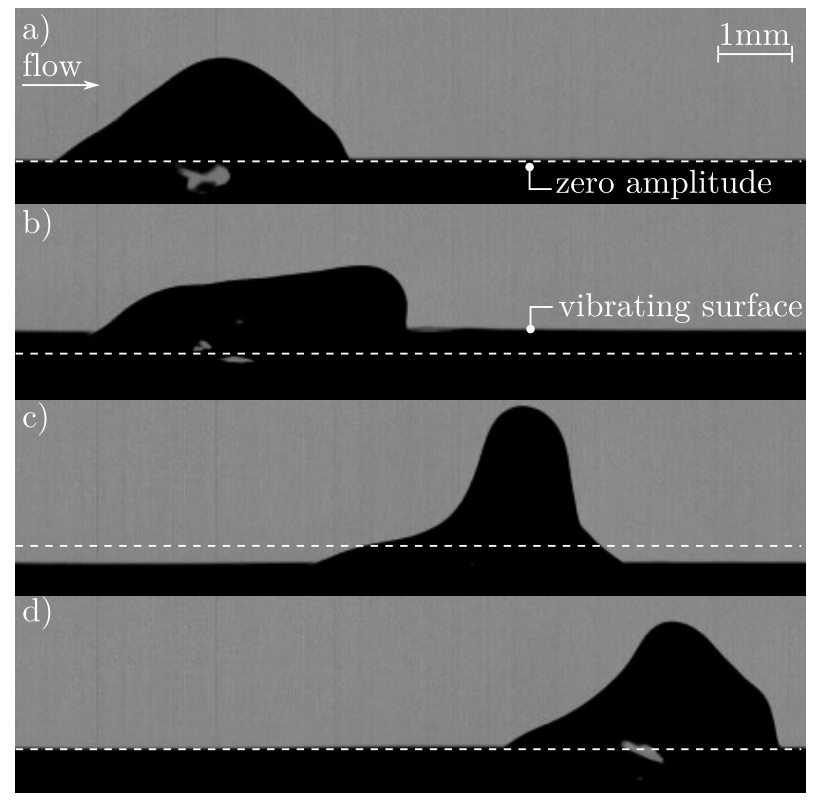

Figure 6: Droplet contour due to superposition of vibration and shear flow. 


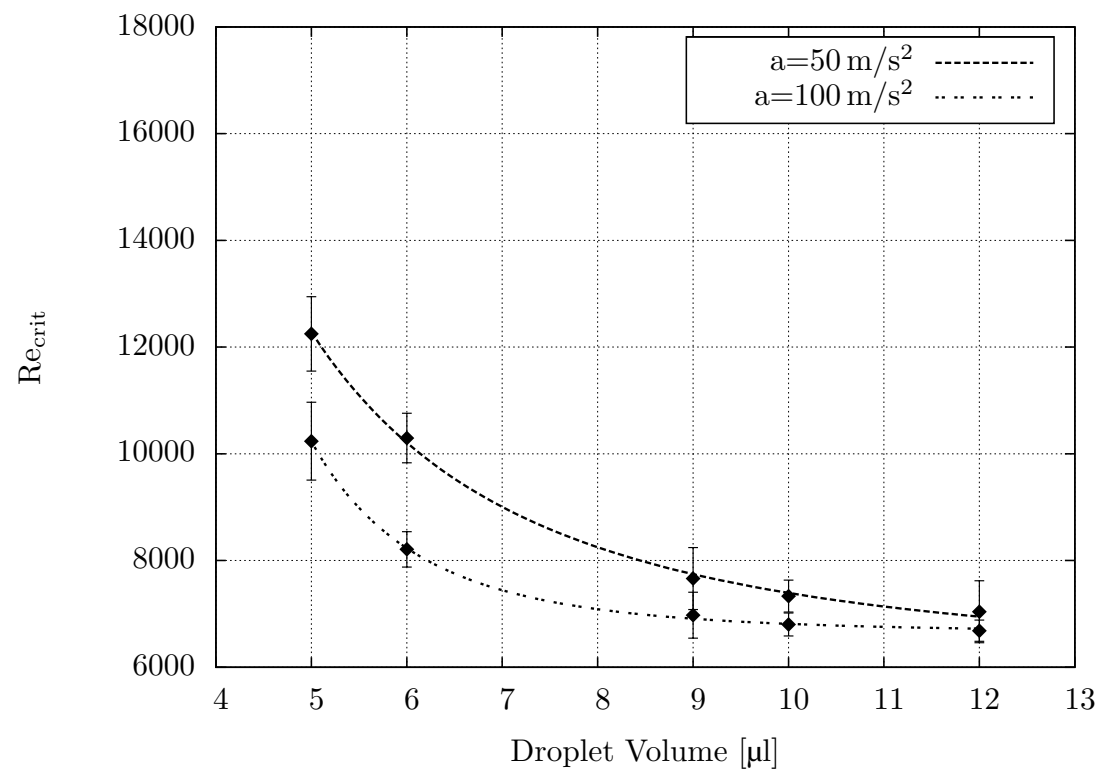

Figure 7: Reynolds numbers for superposition of vibration and shear flow for different drop volumes.

Separate experiments with shear flows indicate that droplet motions are initiated when a critical flow velocity is reached. A forward-backward oscillation is observed before the drop starts to move. The flow velocity required to initiate drop motions decreases for larger drop volume.

The superposition of vibrational acceleration and shear flow leads to an inconvenient droplet contour and motion, the shaking slip motion. Vibrational acceleration of $\mathrm{a}=50 \mathrm{~m} / \mathrm{s}^{2}$ reduces $\mathrm{Re}_{\text {crit }}$ on average by $33 \%$. Increasing the acceleration to $\mathrm{a}=100 \mathrm{~m} / \mathrm{s}^{2}$ leads to an average reduction of the critical Reynolds number by $41 \%$ when compared to the experiments without vibrations. The experiments show that the influence of vibrations on the initial droplet motion becomes stronger with higher droplet volume. Furthermore, the results indicate that a threshold for the critical Reynolds number exists at approximately $\operatorname{Re}_{\text {crit }}=6900$.

This study concludes that the mobility of water droplets on acrylic glass surfaces does increase significantly when a superposition between vibrational forces and shear flows exists. Furthermore shaking slip motion is detected.

\section{References}

[1] Daniel, S. \& Chaudhury, M., Rectified motion of liquid drops on gradient surfaces induced by vibration. Langmuir, 18(9), pp. 3404-3407, 2002. 
[2] Daniel, S., Sircar, S., Gliem, J. \& Chaudhury, M., Ratcheting motion of liquid drops on gradient surfaces. Langmuir, 20(10), pp. 4085-4092, 2004.

[3] Brunet, P., Eggers, J. \& Deegan, R.D., Vibration-induced climbing of drops. Physical Review Letters, 99(14), pp. 144501.1-144501.4, 2007.

[4] Noblin, X., Buguin, A. \& Brochard-Wyart, F., Vibrated sessile drops: Transition between pinned and mobile contact line oscillations. European Physical Journal E, 14(4), pp. 395-404, 2004.

[5] Shastry, A., Case, M.J. \& Bohringer, K.F., Directing droplets using microstructured surfaces. Langmuir, 22(14), pp. 6161-6167, 2006. 18th IEEE International Conference on Micro Electro Mechanical Systems (MEMS), Miami Beach, FL, Jan 30-Feb 03, 2005.

[6] Duncombe, T.A., Erdem, E.Y., Shastry, A., Baskaran, R. \& Boehringer, K.F., Controlling Liquid Drops with Texture Ratchets. Advanced Materials, 24(12), pp. 1545-1550, 2012.

[7] Hao, P., Lv, C., Zhang, X., Yao, Z. \& He, F., Driving liquid droplets on microstructured gradient surface by mechanical vibration. Chemical Engineering Science, 66(10), pp. 2118-2123, 2011.

[8] Mettu, S. \& Chaudhury, M.K., Motion of Liquid Drops on Surfaces Induced by Asymmetric Vibration: Role of Contact Angle Hysteresis. Langmuir, 27(16), pp. 10327-10333, 2011.

[9] Lamb, H., Hydrodynamics. Cambridge University Press: Cambridge, 1932.

[10] Lyubimov, D.V., Lyubimova, T.P. \& Shklyaev, S.V., Non-Axisymmetric Oscillations of a Hemispherical Drop. Fluid Dynamics, 39(6), pp. 851-862, 2004.

[11] Celestini, F. \& Kofman, R., Vibration of submillimeter-size supported droplets. Physical Review E, 73(4, 1), pp. 41602.1-41602.6, 2006.

[12] Moon, J., Kang, B. \& Kim, H., The lowest oscillation mode of a pendant drop. Physics of Fluids, 18(2), 2006.

[13] Al-Hayes, R.A.M. \& Winterton, R.H.S., Bubble diameter on detachment in flowing liquids. International Journal of Heat and Mass Transfer, 24(2), pp. 223-230, 1981.

[14] Duhar, G. \& Colin, C., Dynamics of bubble growth and detachment in a viscous shear flow. Physics of Fluids, 18(7), 2006.

[15] Theodorakakos, A., Ous, T., Gavaises, A., Nouri, J.M., Nikolopoulos, N. \& Yanagihara, H., Dynamics of water droplets detached from porous surfaces of relevance to PEM fuel cells. Journal of Colloid and Interface Science, 300(2), pp. 673-687, 2006.

[16] Durbin, P.A., Considerations on the moving contact-line singularity, with application to frictional drag on a slender drop. Journal of Fluid Mechanics, 197, pp. 157-169, 1988.

[17] Fan, J., Wilson, M.C.T. \& Kapur, N., Displacement of liquid droplets on a surface by a shearing air flow. Journal of Colloid and Interface Science, 356(1), pp. 286-292, 2011. 